\title{
A long distance Laser Altimeter for terrain relative navigation and spacecraft landing
}

\author{
Diego F. Pierrottet \\ Coherent Applications, Inc., 20 Research Drive, Suite 500, Hampton, Virginia, 23666, E-mail: \\ d.f.pierrottet@cailabs.net \\ Farzin Amzajerdian, Bruce Barnes \\ NASA Langley Research Center, Hampton, Virginia 23682
}

\begin{abstract}
A high precision laser altimeter was developed under the Autonomous Landing and Hazard Avoidance (ALHAT) project at NASA Langley Research Center. The laser altimeter provides slant-path range measurements from operational ranges exceeding $30 \mathrm{~km}$ that will be used to support surface-relative state estimation and navigation during planetary descent and precision landing. The altimeter uses an advanced time-of-arrival receiver, which produces multiple signal-return range measurements from tens of kilometers with $5 \mathrm{~cm}$ precision. The transmitter is eye-safe, simplifying operations and testing on earth. The prototype is fully autonomous, and able to withstand the thermal and mechanical stresses experienced during test flights conducted aboard helicopters, fixed-wing aircraft, and Morpheus, a terrestrial rocket-powered vehicle developed by NASA Johnson Space Center. This paper provides an overview of the sensor and presents results obtained during recent field experiments including a helicopter flight test conducted in December 2012 and Morpheus flight tests conducted during March of 2014.
\end{abstract}

Key words: ALHAT, Lidar, range finder, altimeter

\section{INTRODUCTION}

As exploration on the surfaces of small planetary bodies continue, greater demands are placed on the technology driving the Guidance, Navigation, and Control (GN\&C) of the landing vehicle. Entry, descent and landing (EDL) challenges include the removal of the vehicles' kinetic energy in a thin atmosphere, coupled with requirements for precision delivery of payloads to potentially hazardous terrain.

In support of future human or robotic exploration of Near Earth Objects (NEO), Lunar, and Mars missions, development of enhanced GN\&C related technologies that enable the delivery of higher mass vehicles to surfaces at higher altitudes and with greater accuracy are neededi. Accurate estimates of range and descent rate are needed for precision soft landing. NASA Langley Research Center, under the Autonomous Landing and Hazard Avoidance Technology (ALHAT) project is developing advanced, laser-based (lidar) sensors to meet NASA's planetary exploration needs for precise, safe, and soft landing of payloads. ALHAT is comprised of several systems fused for this purpose. These systems include; an Autonomous Flight Manager (AFM), GN\&C algorithms, sensor algorithms for Terrain Relative Navigation (TRN), and commercial off the shelf (COTS) sensors such as IMUs, star trackers and altimeters representing the actual lander sensors that will provide vehicle state and attitude knowledge. The primary function of the Laser Altimeter (LA) described in this paper is to provide information for the implementation of TRN. The LA is a key ALHAT sensor that will measure range to the surface, and compare them to onboard reconnaissance data which will update the onboard navigation state with enough precision and accuracy to maintain minimal position error and dispersion along the descent trajectory. Currently, the performance objectives for the altimeter are met using laser based technology. The receiver provides multiple range measurements from a single laser pulse, suitable for use in high altitude aircraft applications and test flights, where the laser beam may encounter several cloud layers or dust. This paper provides an overview of the sensor and presents results obtained during recent field experiments. 


\section{SENSOR DESCRIPTION}

The LA was built in a compact and rugged housing in order to support ALHAT testing during hot weather, and aboard various high vibration test platforms. Figure 1 shows the dimensions of the prototype sensor. The housing was built around a Nd:YAG laser with an attached OPO to produce pulses at $1574 \mathrm{~nm}$ wavelength. This wavelength is preferred because it is eye-safe at the operational pulse energies, which simplifies the safety logistics during field tests. To accommodate the high ambient temperatures associated with tests sites such as summers in Death Valley, California, or the Nevada Test Site, an air cooled COTS laser was modified with active cooling and control system that maintains the laser temperature constant and thermally stable during ambient temperatures as high as 50-degrees C. The active cooling system requires convective heat removal using forced air, and thus approximately one third of the volume of the altimeter chassis is attributed to the eye-safe laser and the cooling requirements associated with field tests. Future implementations of a space qualified system will not have these requirements, which will allow significant mass and volume reductions to the system. The current system provides a proof of design for key sensor components.
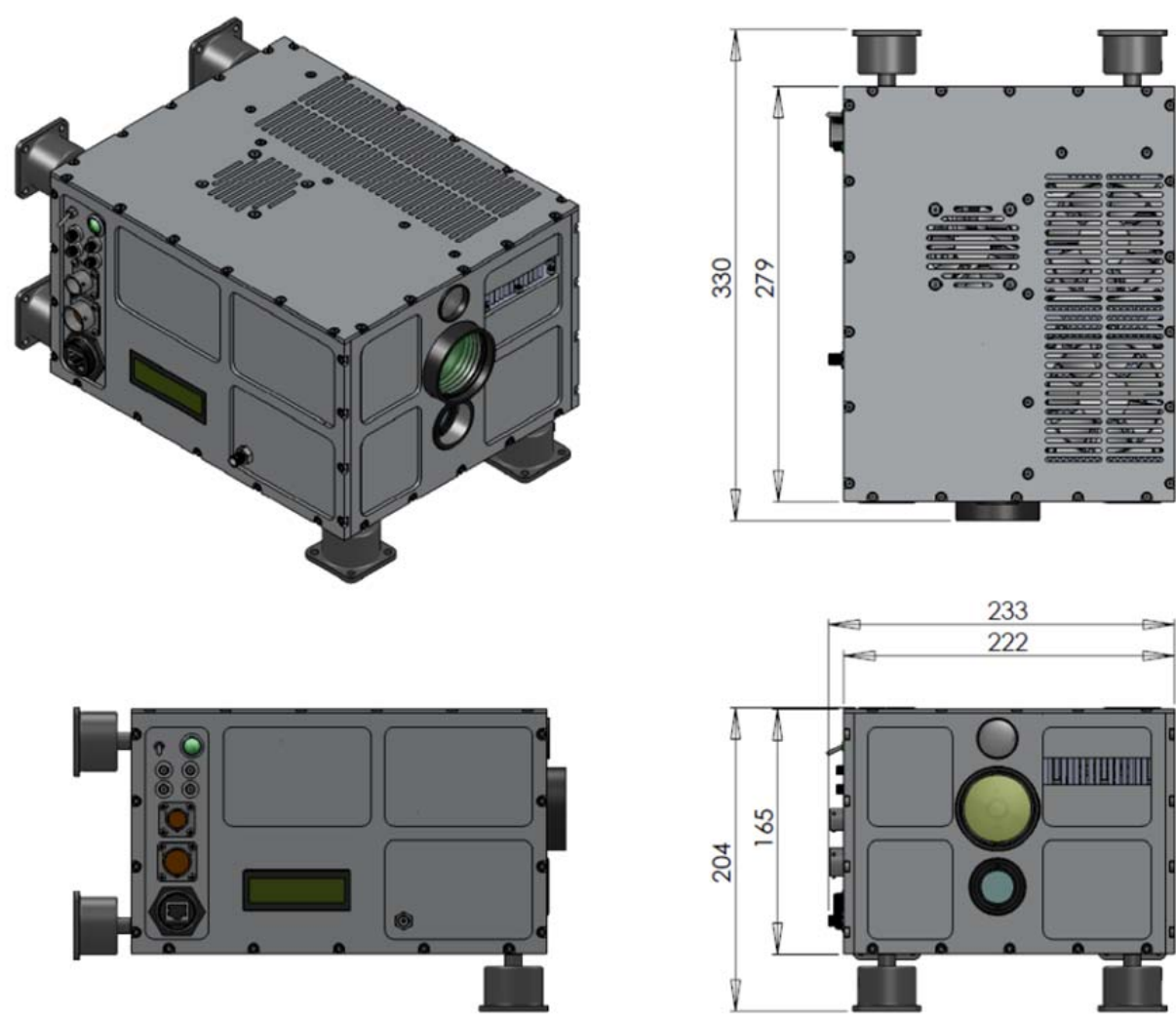

Figure 1. Laser Altimeter chassis showing optical and electronic interfaces.

As seen on Fig. 1, the chassis has several interface connectors and display on the side of the unit. The connectors include output pulse monitor (OPM) for timing synchronization and diagnostics, signal monitor, GPS input (an option used during standalone testing), laser enable interconnect, Ethernet for input command and output telemetry, input power, and status display. On the front of the unit, the receiver lens sits above the smaller transmitter port. Immediately above the receiver lens is a USB bore-sight camera used for convenience in alignment and pointing. The chassis can be mounted on a standard tripod for ground based testing. To mitigate the high vibration environment of helicopters or rocket based Vertical Test Beds (VTB), six vibration isolation mounts are used to attach the sensor to the platform.

The transmitter laser produces pulses of approximately $3 \mathrm{nsec}$, at pulse rate frequencies up to $50 \mathrm{~Hz}$. The pulse is spatially magnified to a collimated output diameter of approximately $30 \mathrm{~mm}$, reducing its beam divergence. The optical receiver 
uses a $50 \mathrm{~mm}$ aperture, placed in a bistatic configuration, meaning the transmitter and the receiver field of view are mounted in parallel to each other. The field of view of this receiver is slightly larger than the transmitter to accommodate spatial pointing jitter of the transmitter. Figure 2 is a diagram describing the configuration of the primary system components.

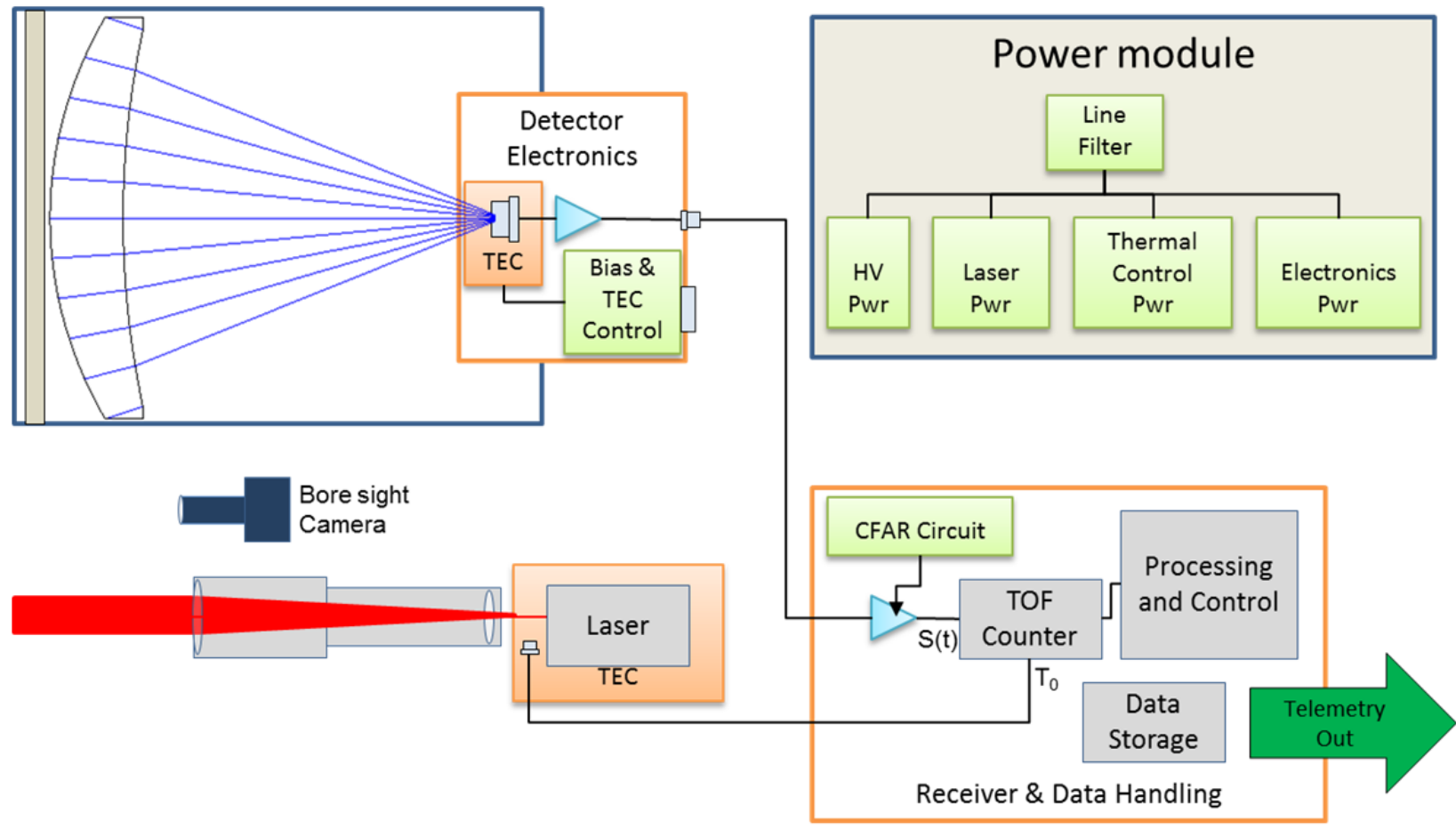

Figure 2. Laser altimeter system architecture block diagram.

The detector is a thermally controlled InGaAs avalanche photo-diode, capable of very high sensitivity and low noise for optimized signal to noise ratio. The detector with its pre-amplifier and signal conditioning circuit are all mounted in a monolithic assembly for electronic and mechanical robustness. The detection circuitry is connected to the electronic receiver module where pulse detection is performed. The receiver includes a constant false alarm rate (CFAR) circuit designed to maintain the false alarm rate to below $2 \%$.

As stated previously, the laser thermal stability is maintained by thermo-electric cooling system. Power for the cooling system, laser, detector bias voltage, and system electronics are generated internally in the Power Module, from a common inline filter of the host vehicle power.

The LA obtains slant range measurement from the ground by measuring the round trip time of flight of a laser pulse. Each line of sight range measurement can directly feed the navigation system of a vehicle and significantly improve vehicle state calculations. The LA is designed to provide range measurements from over $30 \mathrm{~km}$ with $2.5 \mathrm{~cm}$ digitizer precision. An advanced time of flight (TOF) high speed counter was developed that provides a count resolution equivalent to $2.5 \mathrm{~cm}$ range. The counter is initiated by high speed detector monitoring the outgoing pulse of the laser transmitter. Once a signal is detected, the counter bits are latched and a second counter is initiated without losing a single clock cycle. This second counter is identical to counter 1 , and continues to count up until a second signal is detected. At this point, counter 2 bits are latched and counter 1 is again initiated. This "Ping-Pong" architecture can continue to receive many signals from a single laser pulse. Through software, the maximum number of detected signals was set to 3, where the last signal detected is used by the vehicle's GN\&C. After each of the counter bits are latched, the system microprocessor collects the data and converts it into engineering units. The data is stored internally, and is also placed in telemetry packets and sent to the host vehicle. All commands, data processing and external interfacing of the sensor are handled within the chassis by the Command and Data Handing $(\mathrm{C} \& \mathrm{DH})$ unit. During an operational flight, the LA will interface to and operate asynchronously with the navigation 
subsystem. Commands will be received from the navigation subsystem, and output data products and system status are sent to the navigation subsystem. The LA generates data products at $50 \mathrm{~Hz}$ and this data and status are packaged and returned to the navigation system at $5 \mathrm{~Hz}$. Data is time-stamped relative to a pulse-per-second (PPS) signal from the navigation system with one - microsecond precision. All output data is recorded by the navigation subsystem and a subset of this information is transmitted via telemetry during flight for display and analysis to a ground station.

\section{FIELD TESTING}

In preparation of the various ALHAT field tests, the LA was characterized and tested at NASA LaRC, where measurements were taken from the top of the Gantry at a platform height of approximately 60 meters above the ground. NASA LaRC sits on the Hampton Roads peninsula, surrounded by the Chesapeake Bay to the east and the James River to the west. From the Gantry's viewpoint, on a clear day, the Westin Hotel in Virginia Beach can be seen. Figure 3 shows the line of sight distance measured from the Gantry to the Westin by the LA. The measurements show a range measurement precision of $18 \mathrm{~cm}$, and although a truth measurement is not available, the distance is confirmed from satellite images of the area. The data also shows outliers with a 2 meter offset. This offset is due to an intermittent bit error in the counter, which was subsequently repaired.

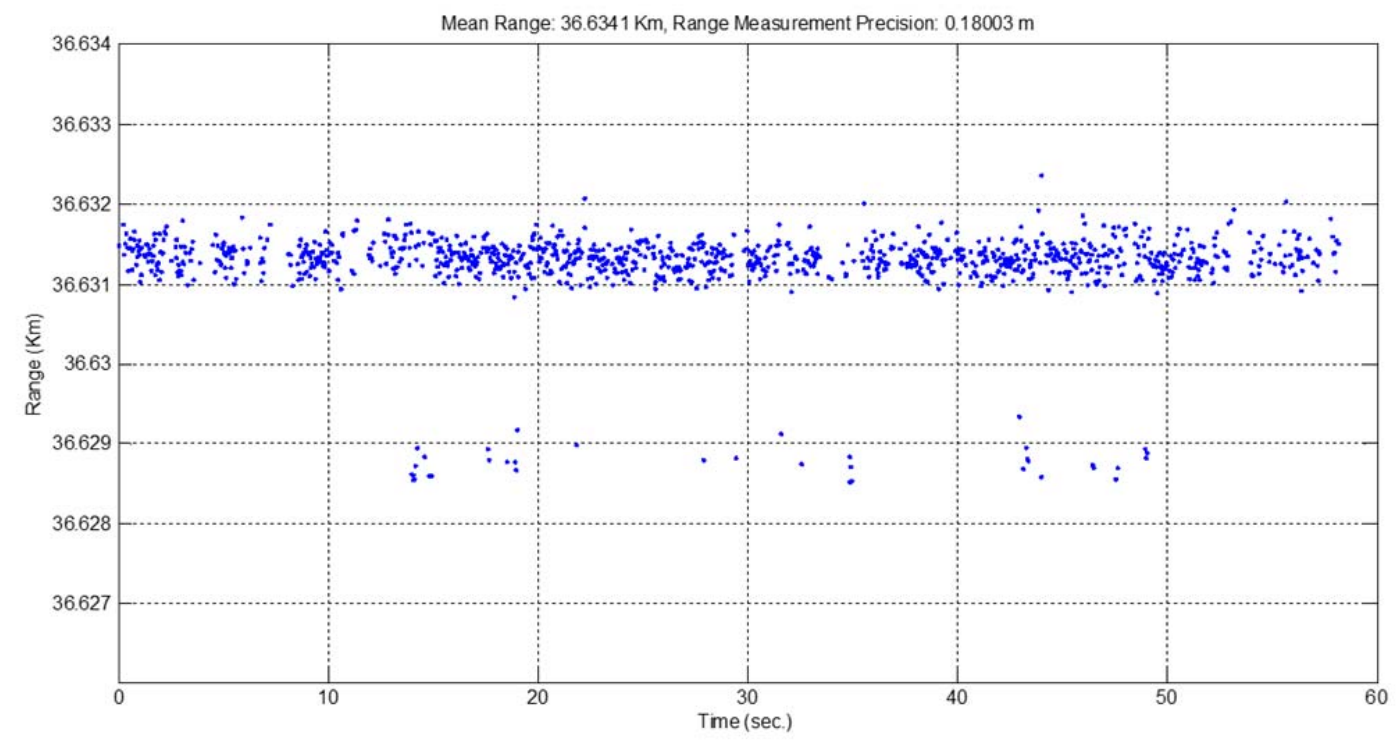

Figure 3. Line of sight range measurements obtained from the Gantry at NASA LaRC, to the Westin in Virginia Beach on a clear day in Hampton Roads.

The laser altimeter has participated in several ALHAT field tests. The first field test was carried out in 2009, where the sensor was mounted on a gimbal inside a Beech B200 Super King airplane, and flown over the Nevada Test Site. The primary purpose of this test was to demonstrate the TRN algorithm developed for the ALHAT system. Figure 4 is a sample of the raw data collected by the altimeter. The repetitive patterns of range correspond to several loops in a race track flight pattern over hills and craters over the desert.

In 2010, ALHAT performed a field test campaign from NASA Dryden, in California. The sensor was configured to collect data together with the 3-dimensional Flash Lidar, and the high precision Navigation Doppler Lidar, mounted in an instrumentation pod which in turn was carried by an Erickson Sky Crane helicopter. This test provided further advances to the three instruments and their role within the ALHAT system. 


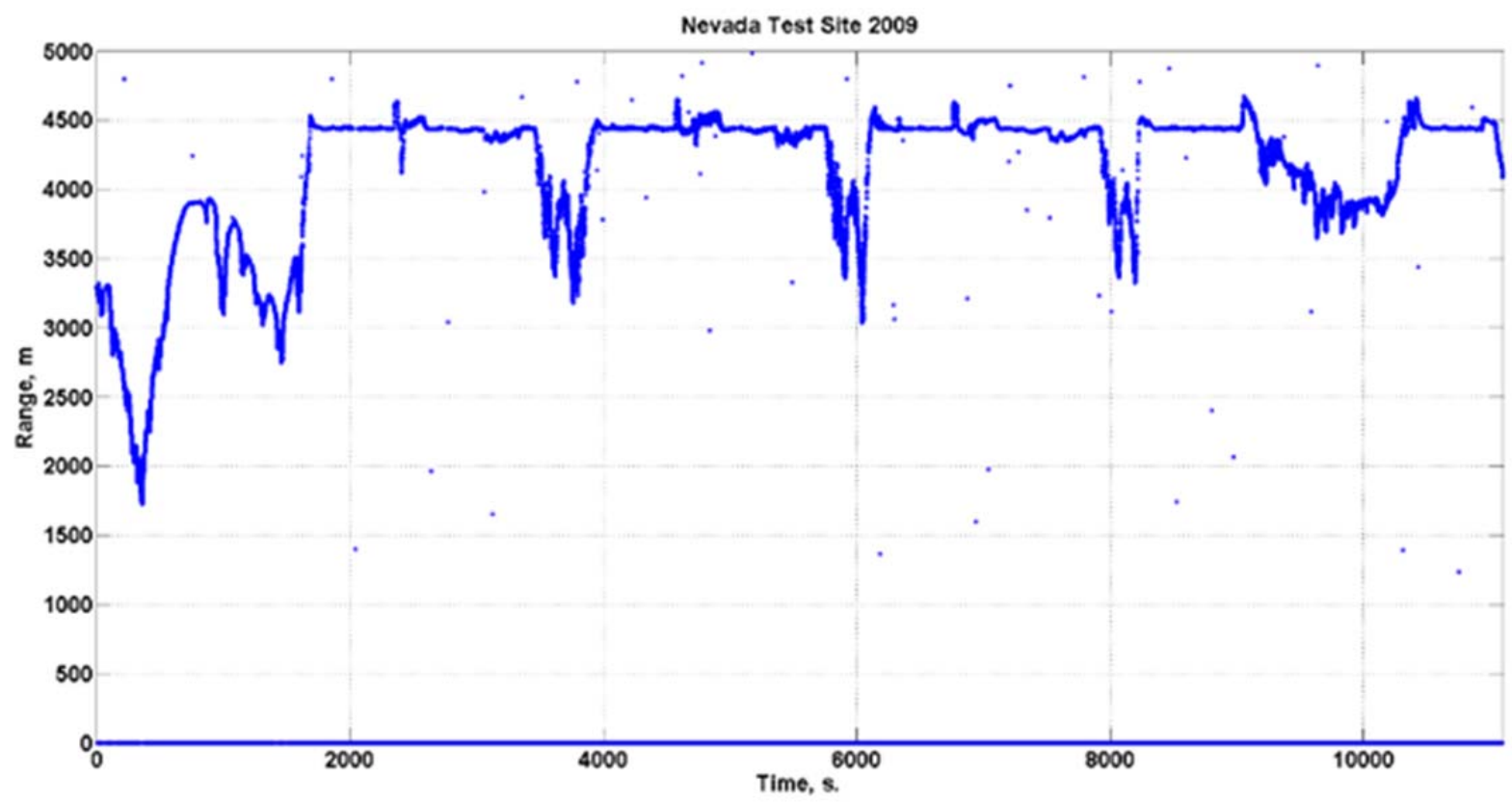

Figure 4. Sample of flight test data from Beech B200 aircraft flown over Nevada Test Site in 2009.

A similar test series was performed in 2012, this time at Kennedy Space Center, where a field was created, complete with rocks and craters, simulating potential hazards around a landing site. For this test, the sensors could operate in an autonomous configuration, with real time telemetry outputs that would be provided to the navigation computer. Figure 5 shows the instruments mounting location underneath NASA's Bell UH-1H Huey test helicopter.

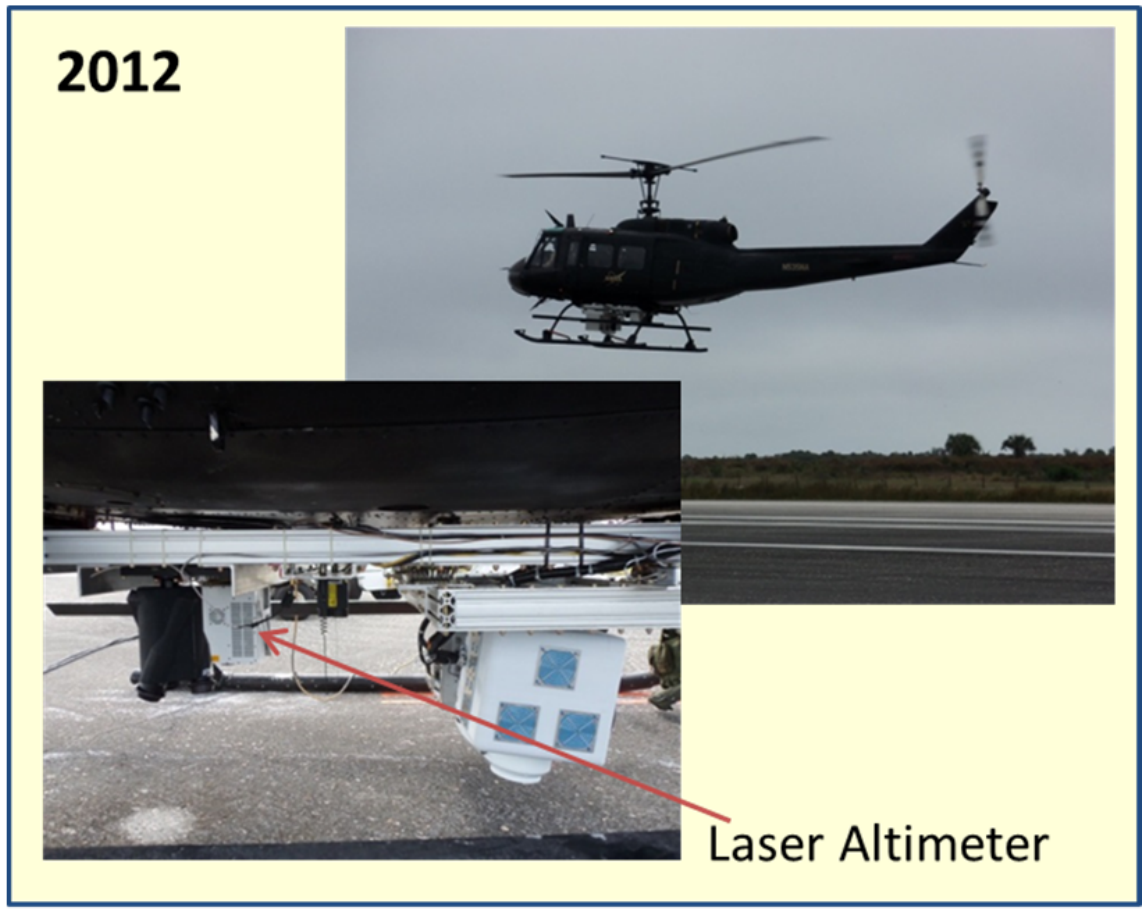

Figure 5 Laser altimeter mounted underneath the helicopter together with the Flash Lidar, and the Navigation Doppler Lidar at Kennedy Space Center. 


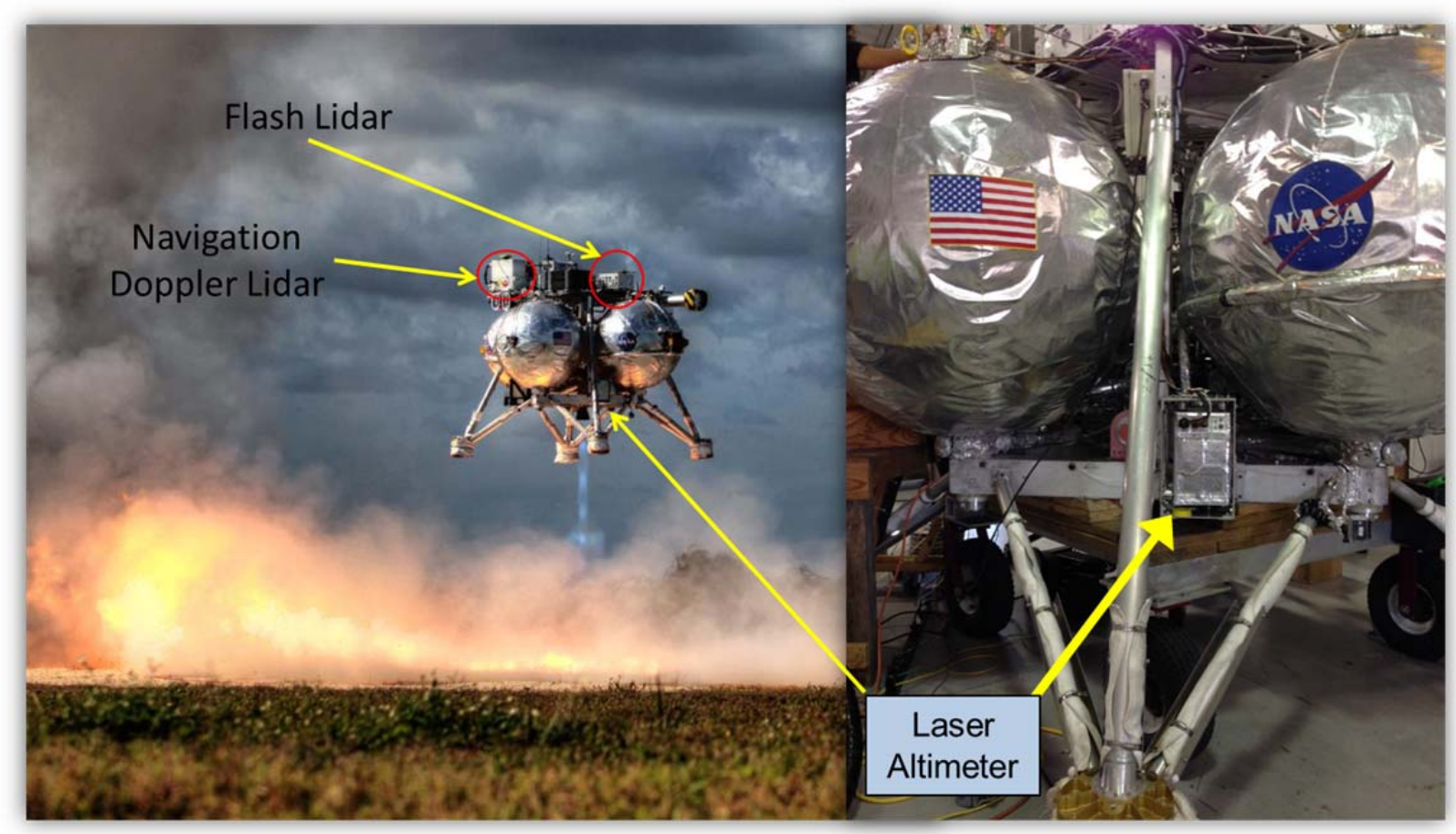

Figure 6 The Morpheus/ALHAT test configuration. The laser altimeter is mounted on the lower support frame of the vehicle.

Finally, the ALHAT sensors are mounted on the Morpheus Vertical Test Bed and operated in a total autonomous configuration, feeding sensor data to the ALHAT navigation computer. The laser altimeter was mounted on the bottom support frame of the vehicle as shown in Fig. 6. It was wrapped with a protective thermal blanket to mitigate the rocket plume temperatures. This field test helps to further advance the ALHAT system, raising the sensor's technology readiness level. At the time of writing this paper, three Morpheus free flights have been performed with the sensors on board in an open loop configuration to the navigation computer. In the coming weeks, two closed loop flights are scheduled to complete the test series.

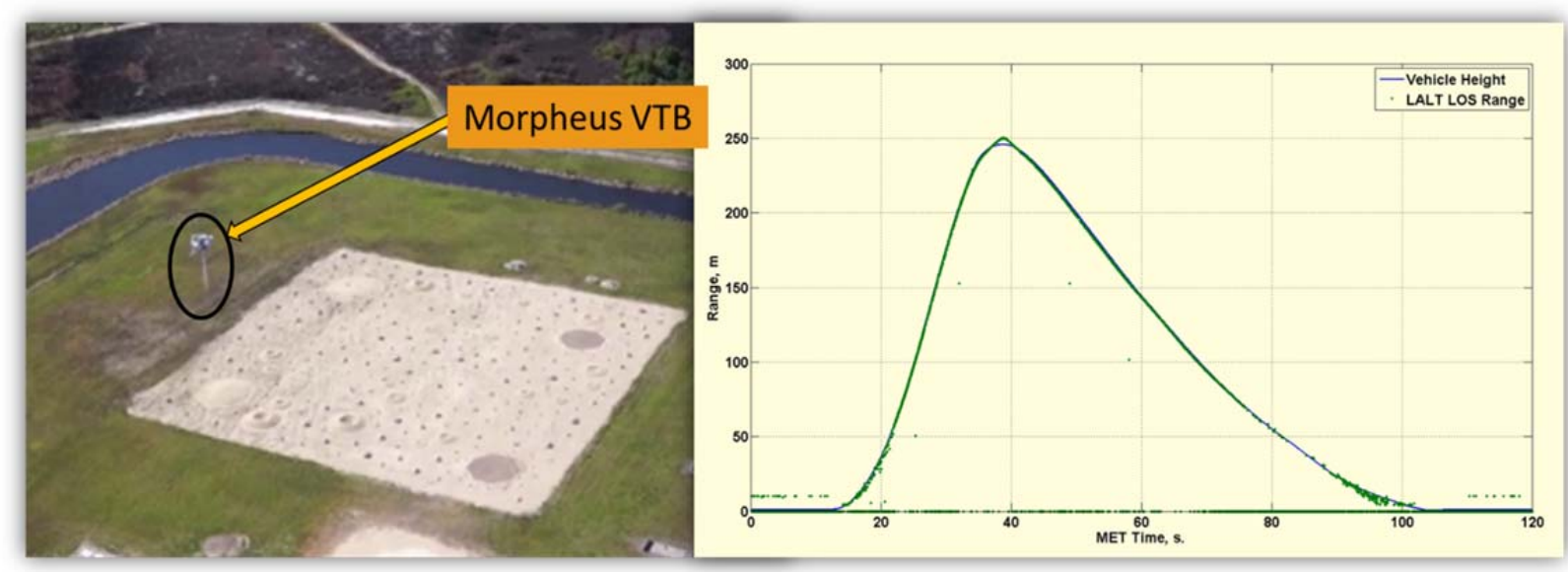

Figure 7 Morpheus/ALHAT field testing at Kennedy Space Center. Altimeter data shown on right 


\section{SUMMARY}

A prototype long distance Laser Altimeter instrument has been developed and tested as a navigation sensor for future space vehicles. This Laser Altimeter is capable of range measurements from over $30 \mathrm{~km}$ with $2.5 \mathrm{~cm}$ resolution. For convenient operation and testing from different platforms, the instrument is designed to be eye-safe and detect multiple returns with a single laser pulse so that the ground signal can be discriminated from signals from clouds or other clutter targets. The rugged and compact design has allowed the laser altimeter to be tested on different platforms: fixed-wing aircraft, helicopter, and a rocket-propelled free-flyer vehicle. The latest tests onboard Morpheus free-flyer vehicle will demonstrate the Laser Altimeter as an integrated sensor of a planetary landing system capable of executing precision navigation to designated target location. Three open-loop flights have already been conducted in preparation for the closed-loop flights scheduled for May 2014. The size, mass, and power of the current prototype system can be further reduced for an actual space flight unit by changing its operational wavelength from eye-safe 1.57 micron to 1.06 micron and simplifying the sensor's thermal management design in accordance with the specific requirements of space environmental.

\section{Acknowledgments}

The authors are grateful to ALHAT project manager, Chirold Epp, NASA Johnson Space Center, for his guidance and support. We also would like to thank NASA's Advanced Exploration Systems (AES) program office for their continued support. The authors also acknowledge the ALHAT and Morpheus team members from NASA Johnson Space Center for facilitating the Morpheus flight tests at NASA Kennedy Space Center.

${ }^{\mathrm{i}}$ Dennehy, C. J. (2010). Present Challenges, Critical Needs, and Future Technological Directions for NASA's GN\&C Engineering Discipline. AIAA Guidance, Navitgation, and Control Conference. Toronto. 


\author{
Filename: If99-17655_final-SPIE_Defense_2014_LALT_paper \\ Directory: $\quad$ C:IUsers $\quad$ Cwbaize\AppDatalLocal $\backslash$ Templ1

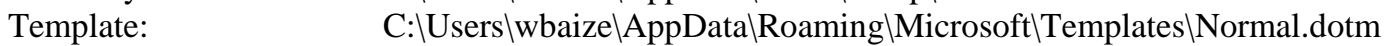 \\ Title: \\ Subject: \\ Author: \\ Pierrottet, Diego F. (LARC-D208)[Coherent Applications, Inc.] \\ Keywords: \\ Comments: \\ Creation Date: $\quad$ 5/12/2014 1:30:00 PM \\ Change Number: $\quad 3$ \\ Last Saved On: $\quad$ 5/12/2014 1:32:00 PM \\ Last Saved By: Amzajerdian, Farzin (LARC-D208) \\ Total Editing Time: 3 Minutes \\ Last Printed On: $\quad$ 5/21/2014 11:16:00 AM \\ As of Last Complete Printing \\ Number of Pages: 7 \\ Number of Words: $\quad 2,294$ (approx.) \\ Number of Characters: $\quad$ 13,079 (approx.)
}

